\title{
Analysis of Lienard II-type oscillator equation by symmetry-transformation methods
}

\section{Özlem Orhan ${ }^{1}$ and Teoman Özer²}

"Correspondence: tozer@itu.edu.tr ${ }^{2}$ Division of Mechanics, Faculty of Civil Engineering, Istanbul Technical University, Maslak, Istanbul, 34469, Turkey

Full list of author information is available at the end of the article

\begin{abstract}
In this study, we consider a Lienard II-type harmonic nonlinear oscillator equation as a nonlinear dynamical system. Firstly, we examine the first integrals in the form $A(t, x) \dot{x}+B(t, x)$, the corresponding exact solutions and the integrating factors. In addition, we analyze other types of the first integrals via the $\lambda$-symmetry approach. We show that the equation can be linearized by means of a nonlocal transformation, the so-called Sundman transformation. Furthermore, using the modified Prelle-Singer approach, we point out that explicit time-independent first integrals can be identified for the Lienard II-type harmonic nonlinear oscillator equation.
\end{abstract}

Keywords: dynamical systems; first integrals; $\lambda$-symmetries; integrating factors; Sundman transformation; Prelle-Singer procedure; Lagrangian and Hamiltonian description

\section{Introduction}

Mathematical modeling of many problems in physics and engineering sciences involve nonlinear ordinary differential equations. Therefore, the methods to solve a nonlinear ordinary differential equation have been continuously developed in the literature; see, for example, [1-8]. The linearization technics deal with obtaining the general solutions of nonlinear equations by using the first integrals and $\lambda$-symmetries. Furthermore, using linearization methods, a nonlinear second-order equation can be converted to a linear second-order ordinary differential equation with known solutions. The first linearization problem for differential equations is solved by Lie [9]. He shows that a second-order ordinary differential equation is linearizable by a change of variables if and only if the equation has the form

$$
\ddot{x}+a_{2}(t, x) \dot{x}^{2}+a_{1}(t, x) \dot{x}+a_{0}(t, x)=0,
$$

where $t$ is the independent variable, $x$ is the dependent variable of the equation, and the over-dot denotes the derivative with respect to $t$ [10].

Moreover, one of these methods is to obtain the general solution by using the first integral of the equation. It is known that some solutions remain invariant under symmetry group transformations; these solutions are called invariant (or similarity) solutions. In

(c) 2016 Orhan and Özer. This article is distributed under the terms of the Creative Commons Attribution 4.0 International License (http://creativecommons.org/licenses/by/4.0/), which permits unrestricted use, distribution, and reproduction in any medium, provided you give appropriate credit to the original author(s) and the source, provide a link to the Creative Commons license, and indicate if changes were made. 
this study, we assume that the ordinary second-order differential equation (1.1) has the first integrals of the form

$$
A(t, x) \dot{x}+B(t, x) .
$$

In order to find the first integrals of the form (1.2), we can use a practicable procedure and apply it to the Lienard II-type harmonic nonlinear oscillator equation. Then it is possible to obtain the first integrals of the form $A(t, x) \dot{x}+B(t, x)$ by using this procedure [11].

From the mathematical point of view, the process of linearization is a difficult task, and it can only be applied to the second-order ordinary differential equations. Therefore, it is necessary to consider other type of transformation techniques for linearization of nonlinear differential equations. In the literature, it is shown that the equations of the form (1.1) can be transformed into the linear equations $X_{T T}=0$ by means of a nonlocal transformation of the form

$$
X=F(t, x), \quad d T=G(t, x) d t,
$$

which is known as the generalized Sundman transformation [12,13]. Such a transformation is also called an $S$-transformation, and the equations that can be linearized by means of an $S$-transformation are called $S$-linearizable [12]. Duarte [14] proves that $S$-linearizable equations must be of the form (1.1). A detailed review for the available generalizations and recent contributions can be found in the references $[15,16]$.

Another method to solve nonlinear differential equations is to obtain $\lambda$-symmetries of the equations. Muriel and Romeo [11] prove that the equations of the form (1.1) have the first integrals of the form (1.2), $\lambda$-symmetries, and the integrating factors $\mu=A(t, x)$. They also show that the equation of the form (1.1) admits $v=\partial_{x}$ for $\lambda$-symmetry of the form [11]

$$
\lambda(t, x, \dot{x})=\alpha(t, x) \dot{x}+\beta(t, x) .
$$

In addition, the modified Prelle-Singer procedure $[17,18]$ is used to apply it to a class of second-order nonlinear ordinary differential equations, to solve several physically interesting nonlinear systems, and to identify a number of important linearization procedures. Prelle and Singer have proposed an algorithmic procedure to find the integrating factor for the system of first-order ordinary differential equations. Once the integrating factor for the equation is determined, it leads to a time-independent integral of motion for the first-order ordinary differential equation. The Prelle-Singer method guarantees that if a first-order ordinary differential equation has a first integral in terms of elementary functions, then this first integral can be found. This method has been generalized to incorporate the integrals with nonelementary functions. Recently, this theory is generalized to obtain general solutions for second- and higher-order ordinary differential equations without any integration [18].

This study is organized as follows. In Section 2, we present some fundamental definitions and theorems. In Section 3, we discuss the nonlinear Lienard II-type harmonic nonlinear oscillator equation and the corresponding linearization methods. Furthermore, the first integral, the $\lambda$-symmetry, the integrating factor, and the transformation pair are pre- 
sented. In Section 4, we apply the modified Prelle-Singer method to the Lienard II-type harmonic nonlinear oscillator equation to obtain Lie symmetries, the first integrals, $\lambda$ symmetries, the integrating factors and the Lagrangian-Hamiltonian functions. The last section summarizes some important results and discussions in the study.

\section{Preliminaries}

\subsection{The first integrals of the form $A(t, x) \dot{x}+B(t, x)$}

In this section, we examine the equations of the form (1.1) that have first integrals of the form $A(t, x) \dot{x}+B(t, x)$ for $A \neq 0$. For this purpose, we introduce the following notations:

$$
\begin{aligned}
& S_{1}(t, x)=a_{1 x}-2 a_{2 t}, \\
& S_{2}(t, x)=\left(a_{0} a_{2}+a_{0 x}\right)_{x}+\left(a_{2 t}-a_{1 x}\right)_{t}+\left(a_{2 t}-a_{1 x}\right) a_{1} .
\end{aligned}
$$

We can say that if $S_{1}=0$, then $S_{2}=0$. Equation (1.1) is $S$-linearizable if and only if $S_{2}=0$. By these definitions we have the following theorem to determine $A(t, x)$ and $B(t, x)$.

Theorem 1 [11] Let us assume that equation (1.1) is S-linearizable, that is, $S_{1}=S_{2}=0$. In addition, let $f(t)$ be the function defined by

$$
f(t)=a_{0} a_{2}+a_{0 x}-\frac{1}{2} a_{1 t}-\frac{1}{4} a_{1}^{2}
$$

and $P=P(t, x)$ be a function such that

$$
P_{t}=\frac{1}{2} a_{1}, \quad P_{x}=a_{2} .
$$

Thus, using equation (2.4), we can determine the function $P=P(t, x)$ explicitly. Similarly, let $g=g(t)$ be a nonzero solution of the linear equation

$$
g^{\prime \prime}(t)+f(t) \cdot g(t)=0,
$$

and $Q=Q(t, x)$ be a function such that

$$
Q_{t}=a_{0} \cdot g \cdot e^{P}, \quad Q_{x}=\left(\frac{1}{2} a_{1}-\frac{g^{\prime}}{g}\right) g \cdot e^{P}
$$

Then, we can obtain the function $Q=Q(t, x)$ from equations (2.5) and (2.6). Finally, the functions $A$ and $B$ are determined as

$$
A=g \cdot e^{P} \quad \text { and } \quad B=Q .
$$

\subsection{The $\lambda$-symmetries and the integrating factors}

Let us consider a second-order ordinary differential equation

$$
\ddot{x}=\Phi(t, x, \dot{x}) .
$$


Then we can say that the vector field $v=\partial_{x}$ is a $\lambda$-symmetry of (2.8) if and only if $\lambda$ is a solution of the equation

$$
\Phi_{x}+\lambda \Phi_{\dot{x}}=\lambda_{t}+\dot{x} \lambda_{x}+\Phi \lambda_{\dot{x}}+\lambda^{2} .
$$

Using the coefficients $a_{0}, a_{1}, a_{2}$ in (1.1), we can easily compute $S_{1}, S_{2}$, and then we can obtain $\lambda$-symmetry for (1.1).

Theorem 2 [11] If $S_{1}=S_{2}=0$, then $\lambda$-symmetry for (1.1) is determined using following feasible algorithm. We consider the equation

$$
h^{\prime}(t)+h^{2}(t)+f(t)=0
$$

where $f(t)$ is defined by (2.3). Then the function $\beta$ is found as

$$
\beta(t, x)=h(t)-\frac{1}{2} a_{1}(t, x) .
$$

Thus, we find that the $\lambda$-symmetry is of the form

$$
\lambda=-a_{2}(t, x) \dot{x}+\beta(t, x) .
$$

Theorem 3 [11] If equation (1.1) has the first integral of the form $I=A(t, x) \dot{x}+B(t, x)$, then equation (1.1) has an integrating factor of the form $\mu=A(t, x)$.

\subsection{The nonlocal transformations}

It is possible to show that the nonlinear second-order equations are linearizable by means of a generalized Sundman transformation. These nonlinear equations are characterized in terms of the coefficients of the equations, and constructive methods to derive the linearizing Sundman transformation can be presented. Thus, the nonlinear ordinary differential equations can be solved by transforming them into the linear ordinary equations whose solutions are known. The nonlocal transformations commonly used in the literature are

$$
X=F(t, x), \quad d T=G(t, x) d t,
$$

called Sundman transformations. These equations are called $S$-linearizable. The secondorder $S$-linearizable equations have first integrals of the form $A(t, x) \dot{x}+B(t, x)$. When a first integral of this form is known, we derive a method to construct the Sundman transformation linearizing the equation. Conversely, if a linearizing Sundman transformation is known, then a first integral of this form is obtained. Now, we present the following theorem that the characterizes $S$-linearizable equations by the coefficients of given differential equations.

Theorem 4 [12] We assume that equation (1.1) is S-linearizable. If $S_{1}=S_{2}=0$ and $\varphi(t)$ is the solution of the equation

$$
\varphi_{t}+\varphi^{2}+f=0
$$


where the function $f(t)$ is defined by (2.3), let $C(t, x)$ be a solution of the following equations:

$$
\begin{aligned}
& C_{t}=a_{0}-C \cdot\left(\frac{a_{1}}{2}+\varphi\right), \\
& C_{x}=\left(\frac{a_{1}}{2}-\varphi\right)-C \cdot a_{2} .
\end{aligned}
$$

If $F(t, x)$ is a solution of the equation

$$
F_{t}=C \cdot F_{x}
$$

and

$$
G=F_{x} \exp \left(-P-\int \varphi(t) d t\right)
$$

then the S-transformation pair F and $G$ is defined by (2.13).

\subsection{Lagrangian and Hamiltonian description}

Assuming the existence of a Hamiltonian

$$
I(x, \dot{x})=H(x, p)=p \dot{x}-L(x, \dot{x}),
$$

where $L(x, \dot{x})$ is the Lagrangian, and $p$ is the canonically conjugate momentum, we have

$$
\frac{\partial I}{\partial \dot{x}}=\frac{\partial H}{\partial \dot{x}}=\frac{\partial p}{\partial \dot{x}} \dot{x}+p-\frac{\partial L}{\partial \dot{x}}=\frac{\partial p}{\partial \dot{x}} \dot{x} .
$$

From equation (2.20) we identify

$$
p=\int \frac{I_{\dot{x}}}{\dot{x}} d \dot{x}+f(x)
$$

where $f(x)$ is an arbitrary function of $x$. Equation (2.21) has also been derived recently by a different methodology. We take $f(x)=0$, and substituting the known expression of $I$ into equation (2.21) and integrating it, we can obtain an expression for the canonical momentum $p$.

\section{The first integral, $\lambda$-symmetry, and the integrating factor of Lienard II-type harmonic nonlinear oscillator equation}

We consider the following Lienard II-type harmonic nonlinear oscillator equation, which possesses an exact periodic solution, exhibiting the characteristic amplitude-dependent frequency of nonlinear oscillator in spite of the sinusoidal nature of the solution of the equation [19]:

$$
\ddot{x}(t)-\frac{2 \dot{x}^{2}(t)}{3 x(t)}+\frac{\omega^{2} x(t)}{3}=0,
$$

where $x$ is the position coordinate, which is a function of time $t$, and $\omega$ is the strength of the forcing, in which these parameters indicate nonlinearity. The Lienard II-type harmonic 
nonlinear oscillator equation has a natural generalization in three dimensions, and these systems can be also quantized exhibiting many interesting features and can be interpreted as an oscillator constrained to move on a three-sphere. In this section, we investigate the first integral of the form $A(t, x) \dot{x}+B(t, x)$ of equation (3.1).

Definition 1 An equation of the form

$$
\ddot{x}+f(x) \dot{x}^{2}+g(x)=0,
$$

where $f(x)$ and $g(x)$ are arbitrary functions of $x$, and over-dots denote differentiation with respect to $t$, is called a quadratic Lienard-type equation. A quadratic Lienard-type equation is linearizable if and only if its coefficients satisfy the condition

$$
g^{\prime}(x)+f(x) \cdot g(x)=\gamma
$$

where $\gamma$ is an arbitrary constant. This condition is called the isochronous condition.

Proposition 1 A dynamical system is called isochronous if it features in its phase space an open, fully-dimensional region where all its solutions are periodic in all its degrees of freedom with the same, fixed, period. In order the Lienard II-type harmonic nonlinear oscillator equation to belong to this class, it must satisfy the isochronous condition to be linearized. Thus, if we apply (3.3) to equation (3.1), then we see that Lienard II-type harmonic nonlinear oscillator equation satisfies the isochronous condition.

Proof Firstly, we compute the function $S_{1}$ for the nonlinear Lienard II-type harmonic nonlinear oscillator equation to classify the equation. The Lienard II-type harmonic nonlinear oscillator equation is of the form (1.1), and the coefficients of (3.1) are given by

$$
a_{2}(t, x)=-\frac{2 \dot{x}^{2}(t)}{3 x(t)}, \quad a_{1}(t, x)=0, \quad a_{0}(t, x)=\frac{\omega^{2} x(t)}{3} .
$$

Using these coefficients, we obtain $S_{1}=0$, which is given by (2.1). Thus, we know from Theorem 1 that $S_{2}$ must be zero if $S_{1}=0$. The function $S_{2}$ computed for the Lienard IItype harmonic nonlinear oscillator equation is found to be zero.

\subsection{The first integral of the form $A(t, x) \dot{x}+B(t, x)$ and the invariant solution}

It can be shown that the Lienard II-type harmonic nonlinear oscillator equation (3.1) has the first integral of the form $A(t, x) \dot{x}+B(t, x)$ by determining the functions $A$ and $B$ using a procedure given above. Then, the equation can be integrated by using this first integral, and the exact solution of the equation can be obtained.

Proposition 2 The Lieanard II-type nonlinear harmonic oscillator equation (3.1) has the first integral of the following form

$$
I=c_{3}-\omega x^{\frac{1}{3}}\left(c_{2} \cos \left(\frac{\omega t}{3}\right)-c_{1} \sin \left(\frac{\omega t}{3}\right)\right)+\frac{\left(c_{1} \cos \left(\frac{\omega t}{3}\right)+c_{2} \sin \left(\frac{\omega t}{3}\right)\right) \dot{x}}{x^{\frac{2}{3}}},
$$

where $c_{1}, c_{2}, c_{3}$ are constants and equation (3.1) has an integrating factor and an invariant solution that corresponds to equation (3.5). 
Proof For this purpose, let a function $P=P(t, x)$ be such that

$$
P_{t}=0, \quad P_{x}=\frac{-2}{3 x} \text {. }
$$

Using (3.6), we obtain the function $P=P(x)$ as

$$
P(x)=\frac{-2 \log x}{3} .
$$

If we compute $f(t)$ using formula (2.3), then we obtain

$$
f(t)=\frac{\omega^{2}}{9}
$$

Let $g=g(t)$ be a nonzero solution of equation (2.5). Then, substituting (3.8) into equation (2.5), we obtain the equation

$$
g^{\prime \prime}(t)+\frac{\omega^{2}}{9} \cdot g(t)=0
$$

the solution of which is

$$
g(t)=c_{1} \cos \left(\frac{\omega t}{3}\right)+c_{2} \sin \left(\frac{\omega t}{3}\right) .
$$

Substituting the functions $P(x)$ and $g(t)$ into (2.6), we obtain the following equations:

$$
\begin{aligned}
Q_{t} & =\frac{1}{3} x^{\frac{1}{3}} \omega^{2}\left(c_{1} \cos \left(\frac{\omega t}{3}\right)+c_{2} \sin \left(\frac{\omega t}{3}\right)\right), \\
Q_{x} & =\frac{-\frac{1}{3} \omega\left(c_{1} \cos \left(\frac{\omega t}{3}\right)+c_{2} \sin \left(\frac{\omega t}{3}\right)\right)}{x^{\frac{2}{3}}} .
\end{aligned}
$$

Solving (3.11)-(3.12), we have

$$
Q(t, x)=c_{3}-\omega x^{\frac{1}{3}}\left(c_{2} \cos \left(\frac{\omega t}{3}\right)-c_{1} \sin \left(\frac{\omega t}{3}\right)\right) .
$$

Substituting this solution into (2.7), we obtain the functions $A(t, x)$ and $B(t, x)$ as follows:

$$
\begin{aligned}
& A(t, x)=\frac{\left(c_{1} \cos \left(\frac{\omega t}{3}\right)+c_{2} \sin \left(\frac{\omega t}{3}\right)\right)}{x^{\frac{2}{3}}}, \\
& B(t, x)=c_{3}-\omega x^{\frac{1}{3}}\left(c_{2} \cos \left(\frac{\omega t}{3}\right)-c_{1} \sin \left(\frac{\omega t}{3}\right)\right) .
\end{aligned}
$$

Thus, the first integral (3.5) is found.

And by Theorem 3 the integrating factor is

$$
\mu=\frac{\left(c_{1} \cos \left(\frac{\omega t}{3}\right)+c_{2} \sin \left(\frac{\omega t}{3}\right)\right)}{x^{\frac{2}{3}}} .
$$


A group-invariant solution of this nonlinear equation can be constructed from the first integral, that is, from (3.5) the invariant solution of equation (3.1) is determined by

$$
x(t)=\frac{\omega c_{1}^{2} c_{5} \cos \left(\frac{\omega t}{3}\right)+\left(c_{4}-c_{3}+\omega c_{1} c_{2} c_{5} \sin \left(\frac{\omega t}{3}\right)\right)^{3}}{\omega^{3} c_{1}^{3}},
$$

where $c_{1}, c_{2}, c_{3}, c_{4}, c_{5}$ are constants.

In the literature, the phase plane method refers to graphically determining the existence of limit cycles in the solutions of the oscillator equations. The solutions to a nonlinear differential equation are a family of functions. Graphically, this can be plotted in the phase plane like a two-dimensional vector field. The vectors representing the derivatives of the points with respect to a parameter time $t$ at representative points are drawn. With enough of these arrows in place, the system behavior over the regions of plane in analysis can be visualized, and the limit cycles can be identified. Then a phase portrait is a geometric representation of the trajectories of a dynamical system in the phase plane. Each set of initial conditions is represented by a different curve or point.

The phase portraits are an invaluable tool in studying dynamical systems. They consist of a plot of typical trajectories in the state space. This reveals information such as whether an attractor, a repeller, or a limit cycle is presented for the chosen parameter value. The concept of topological equivalence is important in classifying the behavior of systems by specifying when two different phase portraits represent the same qualitative dynamic behavior. A phase portrait graph of a dynamical system depicts the system trajectories.

Remark 1 In Figure 1, we see that the solutions of the oscillator equation constitute a limit cycle in the phase plane. The limit cycle begins as a circle and, with varying $\omega$, becomes increasingly sharp.

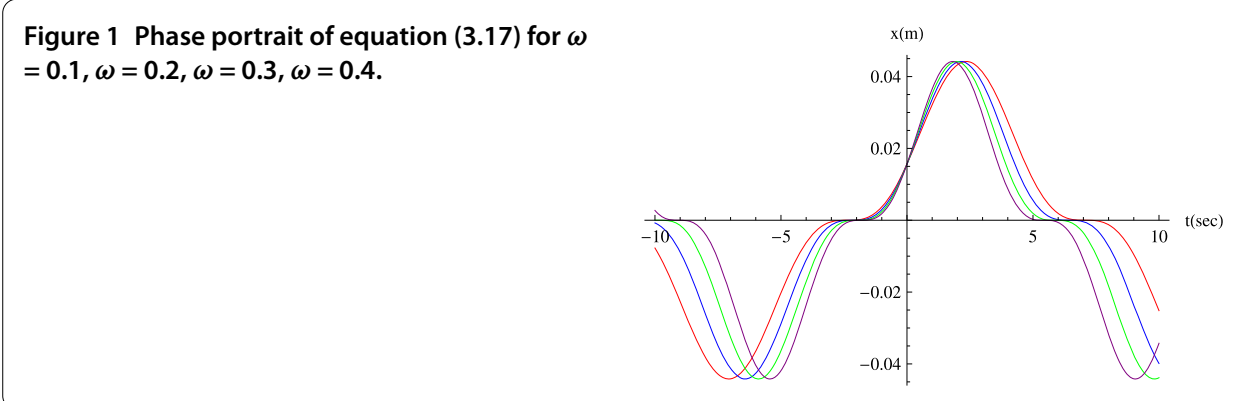

Figure 2 Phase portrait of equation (3.17) for values $x(\mathrm{~m}), \dot{x}(\mathrm{~m} / \mathrm{sec})$, and $\ddot{x}\left(\mathrm{~m} / \mathrm{sec}^{2}\right)$.

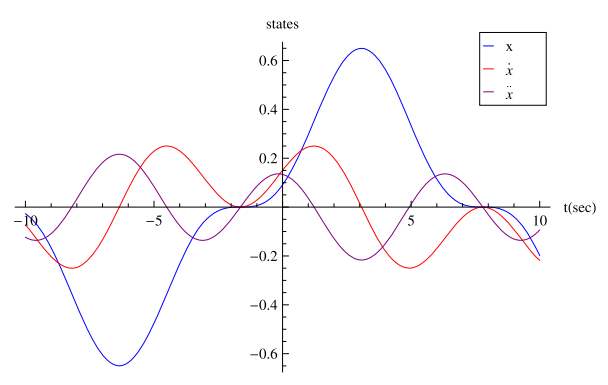


Remark 2 The blue line shows the position $x$ over time, and the red line shows the rate of change $x$ or, in other words, the velocity $\dot{x}$ over time, and the purple line shows the rate of change of the velocity, that is, acceleration, over time in Figure 2. These are the three states of the system simulated over time. The way to interpret this simulation is that if we start the system at $x=0, \dot{x}=0$, and $\ddot{x}=0$ and simulate for 20 seconds, then this is how the system would behave.

Furthermore, we can determine the corresponding Hamiltonian form related to the first integral (3.17). First, the canonical conjugate momentum is

$$
p=\frac{\log (\dot{x})\left(c_{1} \cos \left(\frac{t \omega}{3}\right)+c_{2} \sin \left(\frac{t \omega}{3}\right)\right)}{x^{\frac{2}{3}}},
$$

the Hamiltonian function that corresponds to the canonical conjugate momentum is

$$
H=c_{3}-\omega\left(c_{2} \cos \left(\frac{t \omega}{3}\right)-c_{1} \sin \left(\frac{t \omega}{3}\right)\right) x^{\frac{1}{3}}+\frac{c_{1} \cos \left(\frac{t \omega}{3}\right)+c_{2} \sin \left(\frac{t \omega}{3}\right) \dot{x}}{x^{\frac{2}{3}}} .
$$

Then the corresponding Lagrangian is

$$
L=\frac{\omega\left(c_{2} \cos \left(\frac{t \omega}{3}\right)-c_{1} \sin \left(\frac{t \omega}{3}\right)\right) x+(\log (\dot{x})-1)\left(c_{1} \cos \left(\frac{t \omega}{3}\right)+c_{2} \sin \left(\frac{t \omega}{3}\right)\right) \dot{x}-c_{3} x^{\frac{2}{3}}}{x^{\frac{2}{3}}} .
$$

Now, we can see the graph of the solution. The graph of a dynamical system depicts stable steady states and unstable steady states in the state space. The axes are of state variables. In this case, we deal with the rate of change (velocity) $\dot{x}$ and the rate of change of the velocity (i.e., the acceleration), denoted $\ddot{x}$, as states of the equation.

The graph of (3.17) corresponding to the conjugate momentum (3.18) for four different values $\omega=0.1, \omega=0.3, \omega=0.5$, and $\omega=0.8$ with $\omega$ is shown with four different colors in Figure 3.
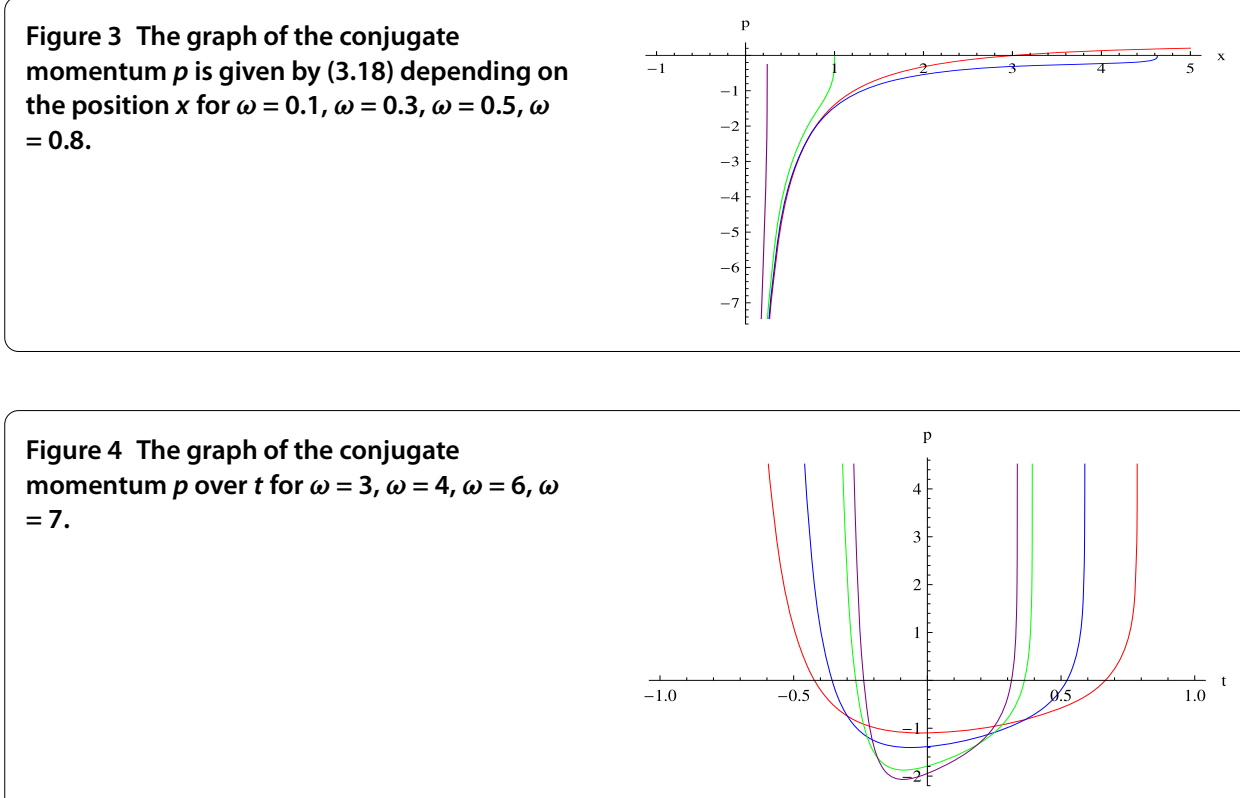
Figure 5 The contour plot for conjugate momentum $p$.

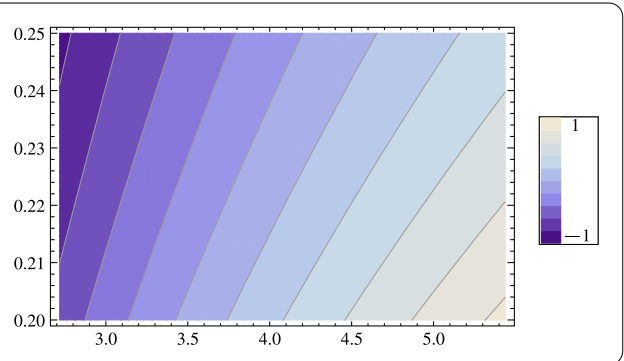

Remark 3 The trajectories are open curves representing unbounded motions in Figure 4.

We can obtain the contour plot graph above for conjugate momentum in Figures 5. Firstly, we find the argument $t$ in terms of $x$ and $\dot{x}$. Using this relation, we can rewrite the canonical conjugate momentum $p$ in terms of $x$ and $\dot{x}$, and we obtain this diagram for different values of $\omega$.

A contour plot is a graphical technique for representing a three-dimensional surface by plotting constant $z$ slices, called contours, on a two-dimensional format. That is, given a value for $z$, lines are drawn for connecting the $(x, y)$ coordinates where that $z$ value occurs. The contour plot is an alternative to a 3-D surface plot. The independent variables are usually restricted to a regular grid. An additional variable may be required to specify the $z$ values for drawing the isolines. If the function does not form a regular grid, you typically need to perform a 2-D interpolation to form a regular grid. The contour plot is used to answer the question 'How does $z$ change as a function of $x$ and $y$ ?'

\subsection{The $\lambda$-symmetry and the nonlocal transformation pair}

We can characterize a second-order ordinary differential equation that can be linearized by means of nonlocal transformations. This characterization is given in terms of the coefficients of the equation and determines the second-order ordinary differential equations that admit $\lambda$-symmetries. There is a systematic method to find $\lambda$-symmetries. These $\lambda$ symmetries can be used to reduce the order of equation. Second-order ordinary differential equations can be integrated by a unified procedure based on $\lambda$-symmetries. An equation of the form (1.1) admits $v=\partial_{x}$ as $\lambda$-symmetries for some function $\lambda$ of the form

$$
\lambda(t, \dot{x}, \ddot{x})=\alpha(t, x) \dot{x}+\beta(t, x)
$$

Proposition 3 We consider equation (3.1) and the functions $S_{1}, S_{2}$ defined by (2.1), (2.2). The condition $S_{1}=S_{2}=0$ is satisfied if and only if $\partial_{x}$ is a $\lambda$-symmetry of (3.1) for $\lambda=$ $\frac{1}{3} \omega \tan \left(\frac{1}{3}\left(-\omega t+9 \omega c_{1}\right)\right)-\frac{2 \dot{x}}{3 x}$.

Proof To obtain a $\lambda$-symmetry of the equation, we first substitute the function $f(t)$ (3.8) into (2.10) and find the following differential equation:

$$
h^{\prime}(t)+h^{2}(t)+\frac{\omega^{2}}{9}=0
$$

Solving this differential equation, we have

$$
h(t)=\frac{1}{3} \omega \tan \left(\frac{1}{3}\left(-\omega t+9 \omega c_{1}\right)\right) .
$$


Substituting the function $h(t)$ into (2.11), we find the function $\beta$ as

$$
\beta(t, x)=\frac{1}{3} \omega \tan \left(\frac{1}{3}\left(-\omega t+9 \omega c_{1}\right)\right)
$$

a $\lambda$-symmetry as

$$
\lambda=\frac{1}{3} \omega \tan \left(\frac{1}{3}\left(-\omega t+9 \omega c_{1}\right)\right)-\frac{2 \dot{x}}{3 x} .
$$

Proposition 4 Equation (3.1) has a transformation pair $F$ and $G$, and the equation can be linearized using this pair. Then the first integral is obtained from this transformation pair.

Proof For given equation (3.1), we know that $S_{1}=0$ and thus $S_{2}=0$. In this situation, we first obtain the transformation pair $F$ and $G$. For this purpose, we consider an algorithm to determine a nonlocal transformation pair of a oscillator equation that is linearizable under a nonlocal transformation. If $\varphi(t)$ is the solution of the equation

$$
\varphi_{t}+\varphi^{2}+\frac{\omega^{2}}{9}=0
$$

then $f(t)$ is computed by (3.8). Solving (3.26), we obtain the function $\varphi(t)$ :

$$
\varphi=\frac{1}{3} \omega \tan \left(\frac{1}{3}(-\omega t+9 \omega)\right) .
$$

Let $C(t, x)$ be a solution of the equations

$$
\begin{aligned}
C_{t} & =\frac{\omega^{2} x}{3}-C \frac{1}{3} \omega \tan \left(\frac{1}{3}(9 \omega-\omega t)\right), \\
C_{x} & =-\frac{1}{3} \omega \tan \left(\frac{1}{3}(9 \omega-\omega t)\right)+\frac{2 C}{3 x} .
\end{aligned}
$$

Solving these equations, we obtain

$$
C(t, x)=x \cdot \omega \tan \left(\frac{1}{3}(\omega t-9 \omega)\right) .
$$

If $F(t, x)$ is a solution of the equation

$$
F_{t}=C \cdot F_{x}
$$

then we obtain the following partial differential equation if we substitute the function $C(t, x)$ into (3.31):

$$
F_{t}-F_{x} x \omega \tan \left(\frac{1}{3}(\omega t-9 \omega)\right)=0 .
$$

Solving this partial differential equation, we find the function $F(t, x)$ :

$$
F(t, x)=\psi\left(x \sec \left(\frac{1}{3}(\omega t-9 \omega)\right)^{3}\right) .
$$


Substituting these functions, we find the function $G$ :

$$
G=x^{\frac{2}{3}} \sec \left(\frac{1}{3}(\omega t-9 \omega)\right)^{4} \psi^{\prime}\left(x \sec \left(\frac{1}{3}(\omega t-9 \omega)\right)^{3}\right)
$$

Then we find an $S$-transformation pair $F$ and $G$ :

$$
\begin{aligned}
& F=\psi\left(x \sec \left(\frac{1}{3}(\omega t-9 \omega)\right)^{3}\right) \\
& G=x^{\frac{2}{3}} \sec \left(\frac{1}{3}(\omega t-9 \omega)\right)^{4} \psi^{\prime}\left(x \sec \left(\frac{1}{3}(\omega t-9 \omega)\right)^{3}\right) .
\end{aligned}
$$

We can integrate the equation using this nonlocal transformation pair.

Now, we can derive the first integral from the transformation pair. Firstly, we find the functions $A(t, x)$ and $B(t, x)$ using the equations

$$
A(t, x)=\frac{F_{x}}{G}, \quad B(t, x)=\frac{F_{t}}{G} .
$$

We obtain the following functions $A(t, x)$ and $B(t, x)$ :

$$
A(t, x)=\frac{\cos \left(3 \omega-\frac{t \omega}{3}\right)}{x^{\frac{2}{3}}}, \quad B(t, x)=x^{\frac{1}{3}} \omega \cos \left(3 \omega-\frac{t \omega}{3}\right) \tan \left(\frac{1}{3}(\omega t-9 \omega)\right) .
$$

The first integral is found using the transformation pair:

$$
I=x^{\frac{1}{3}} \omega \cos \left(3 \omega-\frac{t \omega}{3}\right) \tan \left(\frac{1}{3}(\omega t-9 \omega)\right)+\frac{\cos \left(3 \omega-\frac{t \omega}{3}\right)}{x^{\frac{2}{3}}} \dot{x} .
$$

Now we find the solution of the equation corresponding to this first integral:

$$
\begin{aligned}
x(t)= & \frac{1}{\omega^{3}}\left(c_{1}^{3} \omega^{3} \cos \left(\frac{1}{3}(t-9) \omega\right)^{3}+3 c_{1}^{2} c_{2} \omega^{2} \cos \left(\frac{1}{3}(t-9) \omega\right)^{2} \sin \left(\frac{1}{3}(t-9) \omega\right)\right. \\
& \left.+3 c_{1} c_{2}^{2} \omega \cos \left(\frac{1}{3}(t-9) \omega\right) \sin \left(\frac{1}{3}(t-9) \omega\right)^{2}+c_{2}^{3} \sin \left(\frac{1}{3}(t-9) \omega\right)^{3}\right) .
\end{aligned}
$$

The conjugate momentum corresponding to this solution is given by

$$
p=\frac{\cos \left(\frac{1}{3}(t-9) \omega\right) \log (\dot{x})}{x^{\frac{2}{3}}}
$$

the Lagrangian is

$$
L=\frac{-\omega \sin \left(\frac{1}{3}(t-9) \omega\right) x+\cos \left(\frac{1}{3}(t-9) \omega\right)(\log (\dot{x})-1) \dot{x}}{x^{\frac{2}{3}}},
$$

and, finally, the Hamiltonian function corresponding to the conjugate momentum $p$ is

$$
H=\frac{\omega \sin \left(\frac{1}{3}(t-9) \omega\right) x+\cos \left(\frac{1}{3}(t-9) \omega\right) \dot{x}}{x^{\frac{2}{3}}} .
$$


Figure 6 The graph of the position is given in (3.39) over time $t$ for for $\omega=1, \omega=1.1, \omega=1.2, \omega$ $=1.3$.

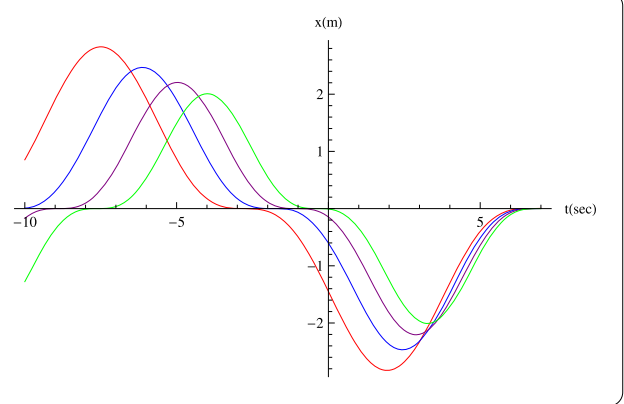

Figure 7 The graphs of the position $x$ is given by (3.39), the velocity $\dot{x}$, and the acceleration $\ddot{x}$ over time $t$ for different values of $\omega$.

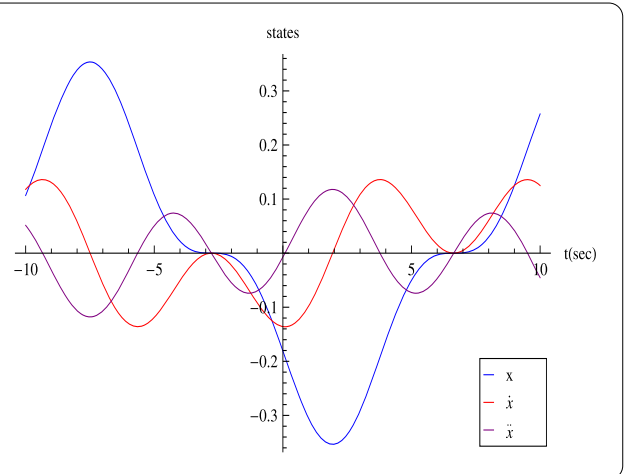

Hence, we can obtain the graphs of these solutions (Figures 6 and 7).

Remark 4 The solution (3.39) of the oscillator equation constitutes a limit cycle in Figure 6. The limit cycle begins as a circle and is changing for different choices of $\omega$.

\section{The extended Prelle-Singer method and $\lambda$-symmetry relation}

In this section, we consider other types of the first integrals and the exact solutions by using the Prelle-Singer method and its relation to $\lambda$-symmetry. This method provides not only the first integrals but also integrating factors. Moreover, we can define the Hamiltonian and Lagrangian forms of the differential equations by using the extended Prelle-Singer method. In this section, we consider the first integrals and exact solutions of the Lienard II-type harmonic nonlinear oscillator equation by the approach related to the PrelleSinger symmetry, $\lambda$-symmetry, and Lie point symmetry as different concepts from the mathematical point of view.

\subsection{The time-independent first integrals}

For the Lienard II-type oscillator equation (3.1), we can write

$$
\phi=\frac{2 \dot{x}^{2}(t)}{3 x(t)}-\frac{\omega^{2} x(t)}{3} .
$$

If this equation has a first integral $I(t, x, \dot{x})=C$ with a constant $C$, then the total differential for the first integral can be written as

$$
d I=I_{t} d t+I_{x} d x+I_{\dot{x}} d \dot{x}=0 .
$$


Substituting equation (4.2) into the formula $\phi d t-d \dot{x}=0$ and adding the null term $S(t, x, \dot{x}) \dot{x} d t-S(t, x, \dot{x}) d x$, we obtain the relation

$$
(\phi+S \dot{x}) d t-S d x-d \dot{x}=0 .
$$

Hence, multiplying (4.2) by the factor $R(t, x, \dot{x})$ (named the integrating factor), we obtain

$$
d I=R(\phi+S \dot{x}) d t-R S d x-R d \dot{x}=0 .
$$

It is clear that equations (4.2) and (4.4) yield the relations

$$
I_{t}=R(\phi+S \dot{x}), \quad I_{x}=-R S, \quad I_{\dot{x}}=-R .
$$

Then, using the compatibility conditions, namely $I_{t x}=I_{x t}, I_{t \dot{x}}=I_{\dot{x} t}, I_{x \dot{x}}=I_{\dot{x} x},(4.5)$ provide us the following system of coupled nonlinear differential equations in terms of $S$, $R$, and $\phi$ :

$$
\begin{aligned}
& S_{t}+\dot{x} S_{x}+\phi S_{\dot{x}}=-\phi_{x}+\phi_{\dot{x}} S+S^{2}, \\
& R_{t}+\dot{x} R_{x}+\phi R_{\dot{x}}=-\left(\phi_{\dot{x}}+S\right) R, \\
& R_{x}-S R_{\dot{x}}-R S_{\dot{x}}=0,
\end{aligned}
$$

where the last equation (4.8) is called the compatibility equation. In addition, wee can determine the first integral $I$ by using the functions $R$ and $S$ with the following relation:

$$
I=r_{1}-r_{2}-\int\left[R+\frac{d}{d \dot{x}}\left(r_{1}-r_{2}\right)\right] d \dot{x},
$$

where

$$
r_{1}=\int R(\phi+\dot{x} s) d t, \quad r_{2}=\int\left(R S+\frac{d}{d x} r_{1}\right) d x .
$$

First of all, we consider the time-independent first integral case, that is, $I_{t}=0$. We can easily find $S$ from the first equation in (4.5):

$$
S=\frac{-\phi}{\dot{x}}=\frac{x \omega^{2}}{3 \dot{x}}-\frac{2 \dot{x}}{3 x}
$$

for $\phi$ in (4.1). Substituting this form of $S$ into equation (4.7), we get

$$
R\left(\frac{2 \dot{x}^{2}+x^{2} \omega^{2}}{3 x \dot{x}}\right)+R_{\dot{x}}\left(\frac{2 \dot{x}^{2}}{3 x}-\frac{x \omega^{2}}{3}\right)+R_{x} \dot{x}+R_{t}=0 .
$$

Equation (4.12) is a first-order linear partial differential equation. To solve this equation, we assume $R$ to be of the form

$$
R=\frac{\dot{x}}{\left(A(x)+B(x) \dot{x}+C(x) \dot{x}^{2}\right)^{r}},
$$


where $A(x), B(x)$, and $C(x)$ are functions of $x$, and $r$ is a constant. If we substitute (4.13) into equation (4.12), then we obtain a set of equations in terms of $\dot{x}$ and its powers. From the solutions of these equations we have

$$
\begin{aligned}
& A(x)=c_{1} x^{\frac{2}{3}+\frac{4}{3 r}} \omega^{2}+c_{3} x^{\frac{4}{3 r}}, \\
& B(x)=c_{2} x^{-\frac{2(r-2)}{3 r}} \\
& C(x)=c_{1} x^{-\frac{4(r-1)}{3 r}}
\end{aligned}
$$

where $c_{1}, c_{2}$, and $c_{3}$ are arbitrary constants. Substituting these functions into equation (4.13), we find

$$
R=\dot{x}\left(c_{3} x^{\frac{4}{3 r}}+c_{1} x^{-\frac{4(r-1)}{3 r}} \dot{x}^{2}+c_{1} x^{\frac{2}{3}+\frac{4}{3 r}} \omega^{2}\right)^{-r},
$$

and substituting the functions $R$ (4.17) and $S$ (4.11) into equations (4.6)-(4.8), we easily check that these equations are satisfied. Thus, we can determine the first integral of the Lienard II-type equation from relation (4.9):

$$
I=\frac{x^{\frac{4(-1+r)}{3 r}\left(x^{\frac{4}{3}\left(-1+\frac{1}{r}\right)}\left(c_{3} x^{\frac{4}{3}}+c_{1}\left(\dot{x}^{2}+x^{2} \omega^{2}\right)\right)\right)^{1-r}}}{2 c_{1}(r-1)},
$$

and, for example, if $r=-2$, then the invariant solution of the Lienard II-type equation is

$$
x(t)=e^{-i\left(t+c_{4} \sqrt{c_{1}}\right) \omega}\left(e^{\frac{2}{3} i\left(t+c_{4} \sqrt{c_{1}}\right) \omega}-c_{3} c_{1} \omega^{2}\right)^{3} .
$$

Furthermore, we can determine the corresponding conjugate momentum related to the first integral (4.18):

$$
p=-\frac{\frac{c_{1}^{2} \dot{x}^{5}}{5}+\frac{2}{3} c_{1} x^{\frac{4}{3}} \dot{x}^{3}\left(c_{3}+c_{1} x^{\frac{2}{3}} \omega^{2}\right)+x^{\frac{8}{3}} \dot{x}\left(c_{3}+c_{1} x^{\frac{2}{3}} \omega^{2}\right)^{2}}{x^{4}} .
$$

Then the corresponding Lagrangian is

$$
\begin{aligned}
L= & -\frac{\dot{x}\left(\frac{c_{1}^{2} \dot{x}^{5}}{5}+\frac{2}{3} c_{1} x^{\frac{4}{3}} \dot{x}^{3}\left(c_{3}+c_{1} x^{\frac{2}{3}} \omega^{2}\right)+x^{\frac{8}{3}} \dot{x}\left(c_{3}+c_{1} x^{\frac{2}{3}} \omega^{2}\right)^{2}\right)}{x^{4}} \\
& +\frac{\left(c_{3} x^{\frac{4}{3}}+c_{1}\left(\dot{x}^{2}+x^{2} \omega^{2}\right)\right)^{3}}{6 c_{1} x^{4}},
\end{aligned}
$$

and the corresponding Hamiltonian form related to the first integral (4.18) is

$$
H=-\frac{\left(c_{3} x^{\frac{4}{3}}+c_{1}\left(\dot{x}^{2}+x^{2} \omega^{2}\right)\right)^{3}}{6 c_{1} x^{4}} .
$$

Thus, we can examine the relation between Hamiltonian function and the position $x$ with the contour plot graph given in Figure 8 . 
Figure 8 The contour plot of Hamiltonian function in terms of $x$ and $\dot{x}$.

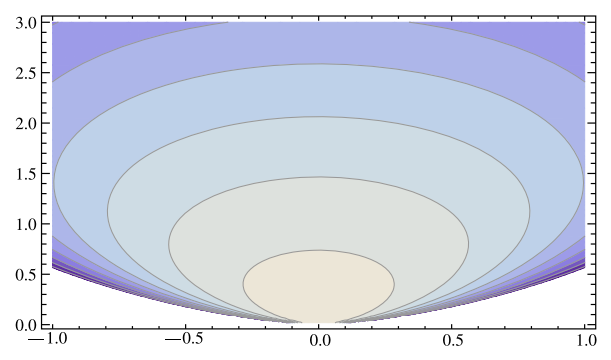

\subsection{The exact solution of the equation using the $\lambda$-symmetries based on a linearization method}

In this section, we examine another method to investigate symmetries of the nonlinear equations. We construct the first integral directly from $\lambda$-symmetry. The procedure essentially involves the following four steps.

1. Find a first integral $w(t, x, \dot{x})$ of $v^{[\lambda,(1)]}$, that is, a particular solution of the equation

$$
w_{x}+\lambda w_{\dot{x}}=0,
$$

where subscripts denote partial derivatives with respect to that variable, and $v^{[\lambda,(1)]}$ is the first-order $\lambda$-prolongation of the vector field $v$.

2. Evaluate $A(w)$ and express $A(w)$ in terms of $(t, w)$ as $A(w)=F(t, w)$, and the operator $A$ is defined in the form

$$
A=\partial_{t}+\dot{x} \partial_{x}+\phi(t, x, \dot{x}) \partial_{\dot{x}}
$$

3. Find a first integral $G$ of $\partial_{t}+F(t, w) \partial_{w}$.

4. Evaluate $I(t, x, \dot{x})=G(t, w(t, x, \dot{x}))$. Then $I(t, x, \dot{x})$ is a first integral, and $\mu(t, x, \dot{x})=I_{\dot{x}}$ is an integrating factor of the given second-order equation.

Now we introduce a first integral and an exact solution of the nonlinear oscillator harmonic equation by using $\lambda$-symmetry (3.25), which is found by linearization method. We first consider the $\lambda$-symmetry (3.25) of the nonlinear oscillator harmonic equation (3.1). The null function $S$ can be written

$$
S=-\lambda=\frac{1}{3} \omega \tan \left(\frac{1}{3}\left(-\omega t+9 \omega c_{1}\right)\right)-\frac{2 \dot{x}}{3 x} .
$$

From (4.23) we have

$$
w=\frac{\dot{x}-x \omega \tan \left(3 \omega-\frac{t \omega}{3}\right)}{x^{\frac{2}{3}}} .
$$

Hence, we can evaluate $A(w)$ as the application of the operator $A(4.24)$ to $w(4.26)$,

$$
A(w)=A=\partial_{t}+\dot{x} \partial_{x}+\left(\frac{\sigma x}{1+\sigma x^{2}} \dot{x}^{2}-\frac{\omega^{2} x}{1+\sigma x^{2}}\right) \partial_{\dot{x}},
$$

and derive $A(w)$ in terms of $(t, w)$ as $A(w)=F(t, w)$, that is,

$$
F(t, w)=-\frac{1}{6} w \omega \sec \left(\frac{1}{3}(t-9) \omega\right) \sin \left(6 \omega-\frac{2 t \omega}{3}\right) .
$$


In the last step, we can find a first integral $G$ of $\partial_{t}+F(t, w) \partial_{w}$ from the first-order partial differential equation of the form

$$
G_{t}+\left(-\frac{1}{6} w \omega \sec \left(\frac{1}{3}(t-9) \omega\right) \sin \left(6 \omega-\frac{2 t \omega}{3}\right)\right) G_{w}=0
$$

the solution of which is

$$
G(t, w)=c_{1}\left(w \cos \left(\frac{1}{3}(t-9) \omega\right)\right)
$$

where $c_{1}$ is an arbitrary constant. Finally, we can express $G(t, w)$ in terms of $(t, x, \dot{x})$ using (4.26) to find the first integral

$$
I=\frac{\cos \left(\frac{1}{3}(t-9) \dot{x}-x \omega \tan \left(3 \omega-\frac{t \omega}{3}\right)\right)}{x^{\frac{2}{3}}} .
$$

The integrating factor can be deduced from the first integral by differentiating it with respect to $\dot{x}$. Thus, we find the integrating factor of the form

$$
\mu=\frac{\cos \left(\frac{1}{3}(t-9) \omega\right)}{x^{\frac{2}{3}}} .
$$

The function $R$ can be written as

$$
R=-\mu=-\frac{\cos \left(\frac{1}{3}(t-9) \omega\right)}{x^{\frac{2}{3}}} .
$$

It is easy to check again that the functions $S$ and $R$ satisfy equations (4.6)-(4.8). Thus, the other exact solution of the Lienard II-type nonlinear harmonic oscillator equation is

$$
x(t)=\frac{\left(c_{1} \omega \cos \left(\frac{1}{3}(t-9) \omega\right)+c \sin \left(\frac{1}{3}(t-9) \omega\right)\right)^{3}}{\omega^{3}},
$$

where $c$ is an arbitrary constant. Now, we see the graph of the corresponding solution to the equation (4.34) in Figure 9. Then, Figure 10 shows the rate of $x(m)$ given by equation (4.34), $\dot{x}(\mathrm{~m} / \mathrm{sec})$, and $\ddot{x}\left(\mathrm{~m} / \mathrm{sec}^{2}\right)$ depending on time $t$.

Furthermore, it is possible to show that we can find other forms of the first integrals and the integrating factors rather then the forms given by (4.31) and (4.32) for the same null function $S$. With this aim, we consider again (4.23) and substitute this form of $S$ into equation (4.7):

$$
\left(R_{t}+\dot{x} R_{x}\right) 3 x+R\left(2 \dot{x}+2 \omega \tan \left(\frac{1}{3}\left(-\omega t+9 \omega c_{1}\right)\right)\right)+R_{\dot{x}}\left(2 \dot{x}^{2}-x^{2} \omega^{2}\right)=0
$$

Equation (4.35) is a first-order linear partial differential equation in terms of $R=R(t, x, \dot{x})$, and it is known that any particular solution is sufficient to construct an integral motion. For this purpose, to seek a particular solution for $R$, we can make a suitable ansatz instead of looking for the general solution by assuming $R$ to be of the form

$$
R=\frac{3 x}{(A(t, x)+B(t, x) \dot{x})^{r}},
$$




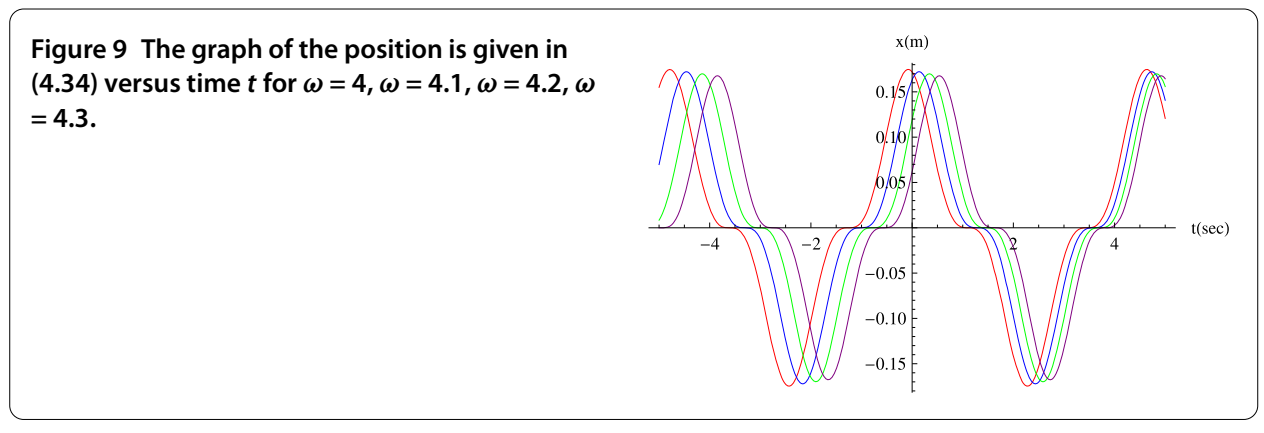

Figure 10 The graphs of the position is given by equation (4.34), the velocity, and the acceleration versus time $t$ for $\omega=4$.

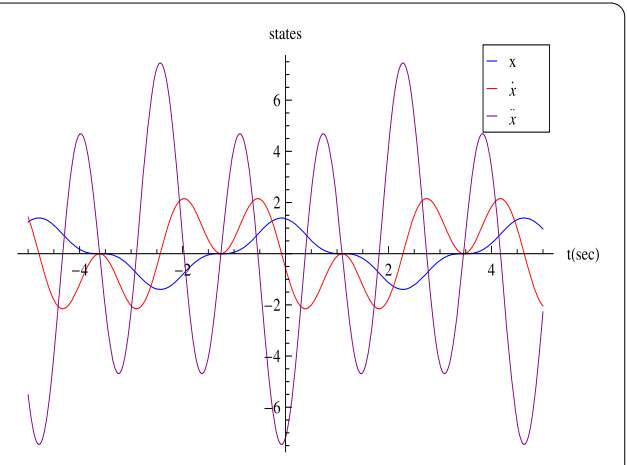

where $A$ and $B$ are functions of their arguments, and $r$ is a constant, which are all to be determined. The denominator of the function $S$ should be the numerator of the function $R$. Since the denominator of $S$ is $3 x$, we fix a numerator of $R$ as $3 x$. Then, substituting (4.36) into (4.35) yields

$$
\begin{aligned}
& -3\left(A(t, x)\left(-5 \dot{x}+x \omega \tan \left(3 \omega-\frac{t \omega}{3}\right)\right)\right. \\
& +B(t, x)\left((-5+2 r) \dot{x}^{2}-r x^{2} \omega^{2}+x \dot{x} \omega \tan \left(3 \omega-\frac{t \omega}{3}\right)\right) \\
& \left.+3 r x\left(A_{t}(t, x)+\dot{x}\left(B_{t}(t, x)+A_{x}(t, x)+\dot{x} B_{x}(t, x)\right)\right)\right)=0 .
\end{aligned}
$$

From the solutions of $A(t, x)$ and $B(t, x)$, the integrating factor $R$ using (4.9), for example, $r=-1$, is written

$$
\begin{aligned}
R & =-\mu \\
& =3 \cos \left(\frac{1}{3}(t-9) \omega\right)\left(c_{1} x^{\frac{2}{3}}+c_{2} \dot{x} \cos \left(\frac{1}{3}(t-9) \omega\right)-c_{2} x \omega \sin \left(3 \omega-\frac{t \omega}{3}\right)\right)
\end{aligned}
$$

and the corresponding time-dependent first integral is

$$
\begin{aligned}
I= & -\frac{3}{4 x^{\frac{4}{3}}}\left(c_{2} \dot{x}^{2}+c_{2} x^{2} \omega^{2}+c_{2}\left(\dot{x}^{2}-x^{2}\right) \cos \left(\frac{2}{3}(t-9) \omega\right)-4 c_{1} x^{\frac{5}{3}} \omega \sin \left(3 \omega-\frac{t \omega}{3}\right)\right. \\
& \left.+4 \cos \left(\frac{1}{3}(t-9) \omega\right)\left(c_{1} x^{\frac{2}{3}} \dot{x}-c_{2} x \dot{x} \omega \sin \left(3 \omega-\frac{t \omega}{3}\right)\right)\right),
\end{aligned}
$$


where $c_{1}$ and $c_{2}$ are arbitrary constants. But it is clear that it is not easy to find an explicit solution for (4.39). Then, we can obtain the Hamiltonian function corresponding to the first integral (4.39), the conjugate momentum is given by

$$
p=-\frac{3 \cos \left(\frac{1}{3}(t-9) \omega\right)\left(x^{\frac{2}{3}} \log (\dot{x})\right)\left(c_{1}-c_{2} x^{\frac{1}{3}} \omega \sin \left(3 \omega-\frac{t \omega}{3}\right)+c_{2} \cos \left(\frac{1}{3}(t-9) \omega\right)\right) \dot{x}}{x^{\frac{4}{3}}},
$$

the corresponding Lagrangian is

$$
\begin{aligned}
L= & \frac{1}{2 x^{\frac{4}{3}}}\left(3 c_{2} x^{2} \omega^{2} \sin \left(\frac{1}{3}(t-9) \omega\right)^{2}-6 c_{1} x^{\frac{5}{3}} \omega \sin \left(3 \omega-\frac{t \omega}{3}\right)\right. \\
& +3 x^{\frac{2}{3}}(\log (\dot{x})-1)\left(-2 c_{1} \cos \left(\frac{1}{3}(t-9) \omega\right)\right) \\
& \left.+c_{2} x^{\frac{1}{3}} \omega \sin \left(6 \omega-\frac{2 t \omega}{3}\right) \dot{x}-3 c_{2} \cos \left(\frac{1}{3}(t-9) \omega\right)^{2} \dot{x}^{2}\right),
\end{aligned}
$$

and the Hamiltonian is

$$
\begin{aligned}
H= & -\frac{3}{2 x^{\frac{4}{3}}}\left(c_{2} x^{2} \omega^{2} \sin \left(\frac{1}{3}(t-9) \omega\right)^{2}-2 c_{1} x^{\frac{5}{3}} \omega \sin \left(3 \omega-\frac{t \omega}{3}\right)\right. \\
& +\dot{x}\left(2 c_{1} x^{\frac{2}{3}} \cos \left(\frac{1}{3}(t-9) \omega\right)-c_{2} x \omega \sin \left(6 \omega-\frac{2 t \omega}{3}\right)\right. \\
& \left.\left.+c_{2} \cos \left(\frac{1}{3}(t-9) \omega\right)^{2} \dot{x}^{2}\right)\right) .
\end{aligned}
$$

\section{Concluding remarks}

The Lienard II-type nonlinear harmonic oscillator equation has a natural generalization in three dimensions and can be interpreted as an oscillator constrained to move on a threesphere. Such a problem is highly nonlinear. In this study, we analyze the first integral of the form $A(t, x) \dot{x}+B(t, x)$, the $\lambda$-symmetries, and the integrating factors of the Lienard IItype nonlinear harmonic oscillator equation, which is a second-order nonlinear ordinary differential equation.

Firstly, we have characterized the second-order nonlinear ordinary differential equations, and this characterization is given by the coefficients of the equation and also determines the first integral, the $\lambda$-symmetry, and the integrating factor. Thus, the Lienard II-type nonlinear harmonic oscillator equation is classified by using functions $S_{1}$ and $S_{2}$, and the first integral of the form $A(t, x) \dot{x}+B(t, x)$ is obtained by an algorithm. Moreover, we presented some properties and characterization of the equation that admits a vector field as $\lambda$-symmetry. Linearization, the symmetries, and the transformation of equations play a crucial role. Furthermore, the nonlinear second-order ordinary differential equations can be linearized by a Sundman transformation. Finally, we apply a Sundman transformation to the Lienard II-type nonlinear harmonic oscillator equation.

We have identified the time-independent first integrals for the Lienard II-type nonlinear harmonic oscillator equation using the modified Prelle-Singer approach. Moreover, we have constructed appropriate Lagrangian and Hamiltonian functions from the time-independent first integrals and transformed the corresponding Hamiltonian forms 
to standard Hamiltonian forms. The important point of the Prelle-Singer procedure lies in finding explicit solutions satisfying all three determining equations (4.6)-(4.8). In our study, we have taken specific ansatz forms to determine the null forms $S$ and the integrating factor $R$. Finally, from our detailed analysis we have shown these results with the phase portraits depending on the choice of parameters, and using these phase portraits, we interpreted geometric meanings of the solutions. Using the Hamiltonian and the conjugate momentum functions, we demonstrated relations among the solutions, Hamiltonians, and conjugate momentum functions by contour plot portraits.

\section{Competing interests}

The authors declare that they have no competing interests.

\section{Authors' contributions}

All authors contributed equally to the manuscript and jointly worked on the results. All authors typed, read, and approved the final manuscript. The authors are very grateful to both reviewers for carefully reading this paper and for their comments.

\section{Author details}

${ }^{1}$ Department of Mathematics Engineering, Faculty of Science and Letters, Istanbul Technical University, Maslak, Istanbul, 34469, Turkey. ${ }^{2}$ Division of Mechanics, Faculty of Civil Engineering, Istanbul Technical University, Maslak, Istanbul, 34469 , Turkey.

Received: 21 October 2015 Accepted: 6 September 2016 Published online: 12 October 2016

\section{References}

1. Noether, E: Invariante Variationsprobleme. Nachr. König. Gesell. Wissen., Göttingen, Math.-Phys. KI. Heft 2(3), $235-257$ (1918) English translation in Transport Theory and Statistical Physics 1, 186-207, 1971.

2. Bluman, GW, Kumei, S: Symmetries and Differential Equations. Springer, New York (1989)

3. Ovsiannikov, LV: Group Analysis of Differential Equations. Nauka, Moscow (1978)

4. Ibragimov, NH (ed.): CRC Handbook of Lie Group Analysis of Differential Equations, vols. I-III (1994)

5. Olver, PJ: Applications of Lie Groups to Differential Equations. Springer, New York (1986)

6. Orhan, Ö, Özer, T: Linearization properties, first integrals, nonlocal transformation for heat transfer equation. Int. J. Mod. Phys. B 30, 1640024 (2016)

7. Özer, T: The group-theoretical analysis of nonlocal Benney equation. Rep. Math. Phys. 60(1), 13-37 (2007)

8. Özer, T: Symmetry group classification for two-dimensional elastodynamics problems in nonlocal elasticity. Int. J. Eng. Sci. 41(18), 2193-2211 (2003)

9. Lie, S: Classification und integration von gewöhnlichen Differentialgleichungen zwischen $x, y$, die eine Gruppe von Transformationen gestatten. III Arch. Math. Naturvidensk. 8(4), 371-458 (1883), Cristinia, see copy of the original book: reprinted of Sophus Lie: Gessammelte Abhandlungen, Band 5, 'Abhandlungen über die Theorie der Transformationsgruppen' S. Lie, Klassifikation und Integration von gewöhnlichen Differentialgleichungen zwischen $x$, y, die eine Gruppe von Transformationen gestatten. I, II, Math. Ann. 32 (1888) 213-281

10. Ibragimov, NH, Magri, F: Geometric proof of Lie's linearization theorem. Nonlinear Dyn. 36, 41-46 (2004)

11. Muriel, C, Romero, JL: Second-order ordinary differential equations and first integrals of the form $A(t, x) \dot{x}+B(t, x)$. J. Nonlinear Math. Phys. 16, 209-222 (2009)

12. Muriel, C, Romero, JL: Nonlocal transformations and linearization of second-order ordinary differential equations. J. Phys. A, Math. Theor. 43, 434025 (2010)

13. Euler, N, Wolf, T, Leach, PGL, Euler, M: Linearisable third-order ordinary differential equations and generalised Sundman transformation: the case $X^{\prime \prime \prime}=0$. Acta Appl. Math. 76, 89-115 (2003)

14. Duarte, LGS, Moreira, IC, Santos, FC: Linearization under non-point transformation. J. Phys. A, Math. Gen. 27, L739-L743 (1994)

15. Chandrasekar, VK, Senthilvelan, M, Lakshmanan, M: On the complete integrability and linearization of certain second-order nonlinear ordinary differential equations. Proc. R. Soc. A 461, 2451-2476 (2005)

16. Chandrasekar, VK, Senthilvelan, M, Lakshmanan, M: A unification in the theory of linearization of second-order nonlinear ordinary differential equations. J. Phys. A, Math. Gen. 39, L69-L76 (2006)

17. Duarte, LGS, Duarte, SES, da Mota, LACP, Skea, JEF: Solving second-order ordinary differential equations by extending the Prelle-Singer method. J. Phys. A, Math. Gen. 34, 3015-3024 (2001)

18. Prelle, M, Singer, M: Elementary first integrals of differential equations. Trans. Am. Math. Soc. 279, $215-229$ (1983)

19. Tiwari, AK, Pandey, SN, Senthilvelan, M, Lakshmanan, M: Classification of Lie point symmetries for quadratic Lienard type equation $x+f(x) x^{2}+g(x)=0$. J. Math. Phys. 54, $053506(2013)$ 